\title{
Cardiac tamponade originating from gastric cancer
}

\author{
Kazuya Hattori · Tadashi Kondo · \\ Masayoshi Yamamoto - Muneaki Watanabe · \\ Hiroaki Satoh
}

Received: 29 June 2009/ Accepted: 30 June 2009/Published online: 22 July 2009

(C) Springer 2009

To the Editor: We previously read with interest the article by Sakai et al. (March 1999) on cardiac tamponade originating from primary gastric signet ring cell carcinoma [1]. We would like to share our experience.

The patient is a 69-year-old male with a history of total gastrectomy for poorly differentiated gastric adenocarcinoma one year earlier at our institution. Although he remained clinically free of disease for 11 months, the patient was seen complaining of feeling weak, tired, short of breath, and bloated for the past few days. On examination, the patient weighed $59 \mathrm{~kg}$, heart rate was 120 , and blood pressure was 100/82 $\mathrm{mm} \mathrm{Hg}$. A paradoxical pulse and jugular venous distension were noted on physical examination. A chest radiograph was performed immediately, which revealed an increased cardio-thoracic ratio and bilateral pleural effusions. These findings suggested cardiac tamponade with evidence of heart failure, and it was confirmed by echocardiography. Pericardiocentesis was performed, and $750 \mathrm{ml}$ of bloody fluid was taken. Cytology confirmed the presence of malignant cells consistent with resected poorly differentiated gastric adenocarcinoma. The patient did not want any additional treatment and was discharged.

Malignant pericardial effusion is a serious and potentially life-threatening illness that requires prompt evaluation, diagnosis, and management. Sakai et al. showed a case of cardiac tamponade originating from primary gastric signet ring cell carcinoma [1]. Kobayashi et al. reviewed clinicopathological studies in 17 patients with cardiac tamponade originating from gastric cancer [2]. In them, 7 patients had signet ring cell carcinoma, 3 had poorly differentiated adenocarcinoma, and 3 had well and moderately differentiated tubular adenocarcinoma [2]. We reported a patient with cardiac tamponade originating from poorly differentiated gastric adenocarcinoma. This condition, therefore, can occur in patients with various types of histological gastric cancers including poorly differentiated adenocarcinoma of the stomach. Practicing physicians must be aware of this condition.

\section{References}

1. Sakai Y, Minouchi K, Ohta H, Annen Y, Sugimoto T. Cardiac tamponade originating from primary gastric signet ring cell carcinoma. J Gastroenterol. 1999;34:250-2.

2. Kobayashi M, Okabayashi T, Okamoto K, Namikawa T, Araki K. Clinicopathological study of cardiac tamponade due to pericardial metastasis originating from gastric cancer. World J Gastroenterol. 2005;11:6899-904.

K. Hattori - T. Kondo · M. Yamamoto · M. Watanabe

H. Satoh $(\bowtie)$

Departments of Internal Medicine and Surgery,

Mito Medical Center, Mito Kyodo General Hospital,

University of Tsukuba, Mito, Ibaraki 310-0015, Japan

e-mail: hirosato@md.tsukuba.ac.jp 